\title{
Chronic Pouchitis Versus Recurrent Crohn's Disease: A Diagnostic Challenge
}

\author{
Christopher R. Weber · David T. Rubin
}

Received: 16 July 2013/Accepted: 18 July 2013/Published online: 8 August 2013

(c) Springer Science+Business Media New York 2013

Up to one-third of patients with ulcerative colitis will require total abdominal colectomy due to being refractory to therapy or the development of mucosal dysplasia or carcinoma. In these patients, the restorative procedure of choice is ileal pouch-anal anastomosis (IPAA), in which the distal ileum is formed into a pouch and anastomosed to the anus. Such a pouch creates a reservoir, which, when functioning properly, will minimize the frequency of bowel movements in the absence of a colon. One frequent complication of IPAA, however, is the development of pouch inflammation, known as pouchitis, which occurs in $15 \%$ of patients in the first post-operative year [6]. The majority of patients with pouchitis are successfully managed with antibiotics, though a portion experience multiple recurrent episodes. Some patients develop inflammation proximal to the pouch or even develop penetrating transmural complications suggesting a diagnosis of Crohn's disease.

Occasionally, patients experience multiple episodes of chronic pouch inflammation due to recurrent Crohn's disease. Although this was previously believed to be due to an initial misdiagnosis of the underlying cause of colitis, more recently, this "transformation" is thought to be due to changing environmental pressure given the altered postsurgical anatomy. Clinically it is important to identify patients with a modification in diagnosis early in order to provide appropriate Crohn's disease therapy in an effort to prevent pouch failure and to eliminate reversion to

C. R. Weber $(\bowtie)$

Department of Pathology, Inflammatory Bowel Disease Center, The University of Chicago Medicine, Chicago, IL 60637, USA e-mail: christopher.weber@uchospitals.edu

D. T. Rubin

Department of Medicine, Inflammatory Bowel Disease Center, The University of Chicago Medicine, Chicago, IL 60637, USA traditional enterocutaneous ileostomies. In order to differentiate between chronic antibiotic-resistant pouchitis, prepouch ileitis, and Crohn's disease, endoscopic examination and biopsies are usually performed. The most important factor to consider is the distribution pattern of inflammation. If the pouch displays features of chronic but nonpenetrating injury, and the pre-pouch ileum and the remainder of the GI tract appear normal, the diagnosis of Crohn's disease is unlikely. Nevertheless, the diagnosis is not always straightforward if the distribution of disease is somewhat patchy, or if there is equivocal evidence of chronic injury in mucosa proximal to the pouch. Under such circumstances, additional diagnostic markers would prove to be quite useful.

One possible histological marker useful in differentiating between Crohn's disease and chronic pouchitis is pyloric gland metaplasia (PGM) [5]. PGM occurs when intestinal-type epithelium, which is normally present in the ileal pouch, is replaced by glands that resemble gastric pyloric glands (Fig. 1b). These metaplastic pyloric glands are easily identified by routine hematoxylin and eosin (H\&E) staining as tall columnar cells with pale pink cytoplasm and small dark basally situated nuclei (Fig. 1a, inset). As the glands develop, they normally grow into a clustered architecture at the mucosal base. PGM may be observed in a number of intestinal disorders associated with repetitive ulcerations and regeneration [4]. PGM is present in the majority of ileal biopsies from patients with Crohn's disease [3], however, since PGM arises in response to recurrent ulceration, it has been considered a non-specific marker. Other causes of PGM include tumors, radiation enteritis, peptic ulceration, tuberculosis, and Yersinia enterocolitis [4]. Nonetheless, these disease manifestations are not typically observed in an ileoanal pouch, and can usually be excluded on clinical grounds [3]. 


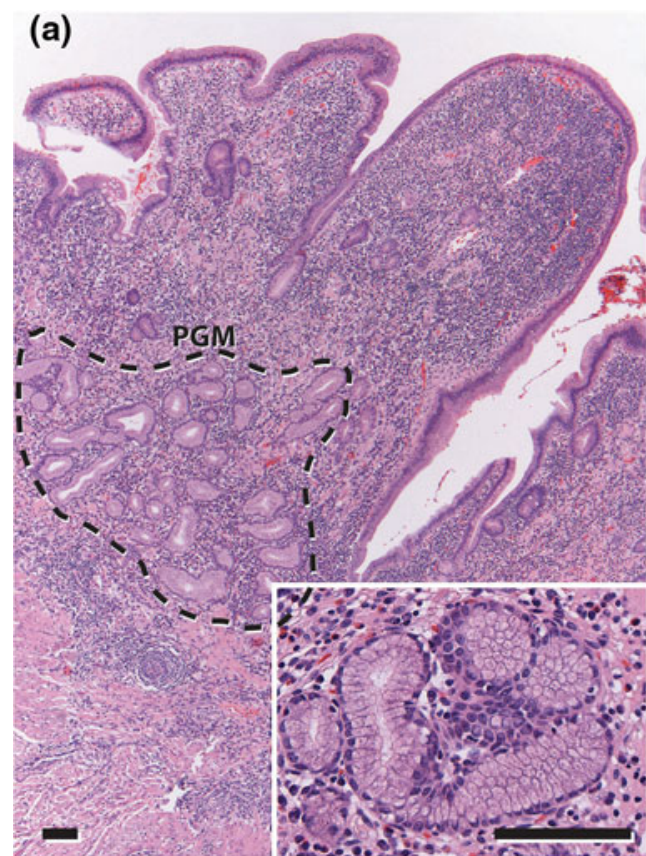

Fig. 1 Pyloric gland metaplasia is a marker of chronic injury in the intestine. a Ileal mucosa from a patient with Crohn's disease demonstrates chronic colitis with villous architectural distortion, basal lymphoplasmacytic infiltrate, and pyloric gland metaplasia at

Therefore, in the appropriate clinical setting, the presence or absence of PGM may contribute to the diagnosis of Crohn's disease. Nevertheless, this has been controversial since PGM also may be associated with chronic antibiotic resistant pouchitis [2].

In the current issue, Agarwal et al. [1] considers antiSaccharomyces cerevisiae antibody (ASCA) in addition to PGM as potential markers to diagnose $\mathrm{CD}$ in patients after IPAA originally diagnosed with ulcerative colitis. Of the 39 patients enrolled in the study, 17 had no history of pouchitis, 9 were diagnosed with chronic pouchitis, and 13 had their diagnoses changed to Crohn's disease based on all available clinical, endoscopic, and histological information. The authors demonstrated a trend towards higher ASCA titers in patients with post-operative complications, although ASCA was not significantly higher in patients with Crohn's disease. For PGM, in patients without a history of pouchitis, the incidence of PGM was $12 \%$; patients with chronic pouchitis had a PGM incidence of $22 \%$, whereas patients subsequently diagnosed with Crohn's disease had a significantly increased incidence of PGM $(77 \%)$. Since PGM may be a marker of chronic disease, one potentially confounding variable was that the duration of the pouch may principally determine the prevalence of PGM, though this possibility was not borne out by the authors' analysis.

The data summarized in Table 1 demonstrate that PGM is a reasonably sensitive and specific marker for Crohn's disease in the pouch. Even though this is a relatively small

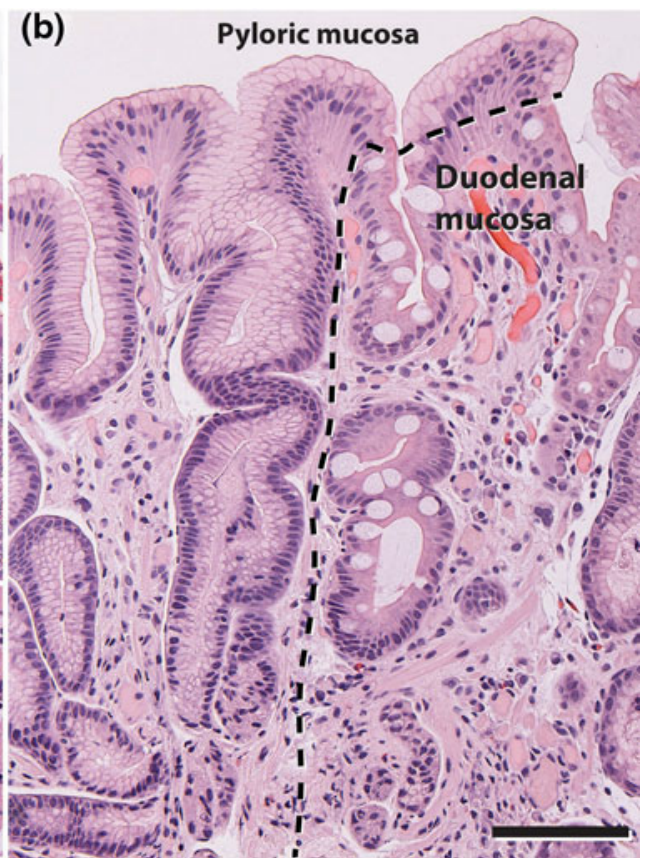

the mucosal base. Pyloric gland metaplasia resembles normal pyloric mucosa. b Gastroduodenal junctional mucosa demonstrates pyloric mucosa on the left and duodenal, intestinal type mucosa on the right. Scale bars $100 \mu \mathrm{m}$

Table 1 Pyloric gland metaplasia is frequently present in pouches of Crohn's disease patients and is usually absent in patients without Crohn's disease [1]

\begin{tabular}{|c|c|c|c|}
\hline & \multicolumn{2}{|c|}{$\begin{array}{l}\text { Patients' status post IPAA with } \\
\text { chronic pouch inflammation }\end{array}$} & \multirow{2}{*}{$\begin{array}{l}\text { Patients' } \\
\text { status post } \\
\text { IPAA without } \\
\text { pouchitis } \\
\text { Negative for } \\
\text { Crohn's }\end{array}$} \\
\hline & $\begin{array}{l}\text { Positive for } \\
\text { Crohn's }\end{array}$ & $\begin{array}{l}\text { Negative for } \\
\text { Crohn's }\end{array}$ & \\
\hline PGM present & 10 & 2 & 2 \\
\hline PGM absent & 3 & 7 & 15 \\
\hline
\end{tabular}

Sensitivity $=10 / 13=77 \%$

Specificity $=7 / 9=78 \%$

False-positive rate $=1-(7 / 9)=22 \%$

False-negative rate $=1-(10 / 13)=23 \%$

Likelihood ratio positive $=$ sensitivity $/(1-$ specificity $)=3.46$

Likelihood ratio negative $=(1-$ specificity $) /$ sensitivity $=0.29$

retrospective study, the data argue that the presence or absence of PGM in pouch biopsies is helpful, and therefore, the pathologist should report the presence or absence of PGM in all pouch biopsies performed on patients with inflammatory bowel disease. Yet, the presence or absence of PGM should not be considered diagnostic, since if it were considered on its own its presence would incorrectly diagnose Crohn's in $22-23 \%$ of patients.

A model that incorporates the family history of Crohn's disease and genetic markers in addition to the presence of 
PGM may further improve the diagnostic accuracy for Crohn's disease of the pouch [7, 8]. Moreover, further clarification of sampling methods and diagnostic yield as well as controlling for intra- and inter-observer variability among pathologists will be necessary for further expansion of these observations.

In summary, the management of chronic pouch inflammation in patients with ulcerative colitis can be challenging. The frequent clinical question, which will guide medical therapy versus possible surgical management, is whether the patient has Crohn's disease. Although usually based on disease distribution, in some cases the diagnosis is not straightforward. The article by Agarwal et al. [1] in this issue shows that the presence of PGM correlates with Crohn's disease of the pouch, providing much-needed additional diagnostic information. Future efforts to refine these observations and correlate them with treatment response will be most helpful.

\section{References}

1. Agarwal S, Stucchi AF, Dendrinos K, et al. Is pyloric gland metaplasia in ileal pouch biopsies a marker for Crohn's disease? Dig Dis Sci. (Epub ahead of print). doi:10.1007/s10620-0132655-4.
2. Kariv R, Plesec TP, Gaffney K, et al. Pyloric gland metaplasia and pouchitis in patients with ileal pouch-anal anastomoses. Aliment Pharmacol Ther. 2010;31:862-873.

3. Koukoulis GK, Ke Y, Henley JD, Cummings OW. Detection of pyloric metaplasia may improve the biopsy diagnosis of Crohn's ileitis. J Clin Gastroenterol. 2002;34:141-143.

4. Lee FD. Pyloric metaplasia in the small intestine. J Pathol Bacteriol. 1964;87:267-277.

5. Liber AF. Aberrant pyloric glands in regional ileitis. AMA Arch Pathol. 1951;51:205-212.

6. Mahadevan U, Sandborn WJ. Diagnosis and management of pouchitis. Gastroenterology. 2003;124:1636-1650.

7. Melmed GY, Fleshner PR, Bardakcioglu O, et al. Family history and serology predict Crohn's disease after ileal pouch-anal anastomosis for ulcerative colitis. Dis Colon Rectum. 2008;51:100-108.

8. Sehgal R, Berg A, Hegarty JP, et al. NOD2/CARD15 mutations correlate with severe pouchitis after ileal pouch-anal anastomosis. Dis Colon Rectum. 2010;53:1487-1494. 